\section{Une étape vers \\ la restauration \\ de la tolérance \\ immunitaire au soi \\ dans les maladies \\ auto-immunes \\ humaines}

\section{Lucienne Chatenoud}

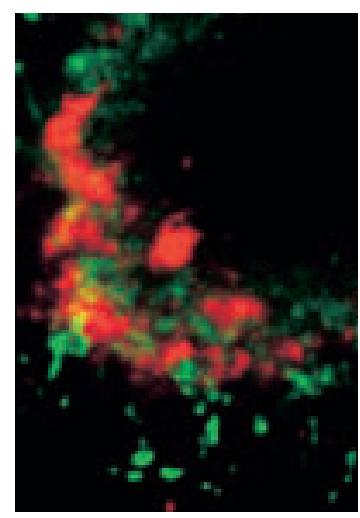

Inserm U580,

Faculté René-Descartes

Paris 5, Hôpital Necker-

Enfants-Malades,

161 , rue de Sèvres

75015 , Paris, France.

chatenoud@necker.fr «reprogrammer» le système immunitaire afin de restaurer la tolérance vis-à-vis des autoantigènes des cellules $\beta$ pancréatiques. Le traitement par anticorps anti-CD3, dont l'efficacité a initialement été démontrée chez la souris, a récemment fait l'objet d'études cliniques qui ont fourni des résultats très encourageants. Les anticorps anti-CD3 pourraient représenter une nouvelle catégorie de médicaments permettant de contrôler à long terme des réactions auto-immunes pathogènes, tout en préservant la capacité de l'hôte à réagir vis-à-vis d'antigènes exogènes. <

\section{La tolérance immunitaire}

L'introduction d'une molécule étrangère (I'antigène) dans l'organisme induit habituellement une réponse immunitaire à la fois cellulaire et humorale pour peu que la molécule ait une taille et une diversité conformationnelle suffisantes. Il est des situations, néanmoins, où l'administration d'un antigène dans certaines conditions, sur lesquelles nous reviendrons, n'induit pas de réponse immunitaire et, de surcroît, produit un état réfractaire qui empêchera l'individu de développer une réponse immunitaire contre ce même antigène si celui-ci lui est ultérieurement présenté de manière immunogène. De façon remarquable, cet état réfractaire, que l'on peut en quelque sorte assimiler à une paralysie immunitaire, est spécifique de l'antigène en question. On le définit comme un état de tolérance immunitaire, et l'antigène qui l'induit est dénommé le «tolérogène ».

De multiples conditions peuvent favoriser l'induction d'une tolérance plutôt que d'une réponse immunitaire. L'âge du receveur, et plus généralement son niveau d'immunocompétence, sont des éléments déterminants. Les souris nouveaunés, dont le système immunitaire est encore immature, sont particulièrement sensibles à l'induction de la tolérance. C'est d'ailleurs dans ce contexte que Peter Medawar réalisa, dans les années 1950, les expériences qui lui valurent le Prix Nobel. L'administration à des souriceaux nouveau-nés de cellules allogéniques (exprimant des antigènes d'histocompatibilité distincts de ceux de l'hôte), en l'absence de tout autre traitement immunosuppresseur, induit un état de tolérance qui se manifeste par le fait que ces souris, une

Article reçu le 14 mars 2006, accepté le 12 juillet 2006. 
fois adultes, ne rejettent pas les allogreffes de peau provenant de donneurs totalement identiques (ou histocompatibles) avec le donneur des cellules injectées à la naissance [1, 2]. Ces observations pionnières ont été suivies par de nombreux travaux visant à recréer, chez l'individu adulte, un statut immunitaire propice, comme celui du nouveau-né, à l'établissement d'une tolérance immunitaire. II s'est avéré qu'une administration de courte durée de certains immunosuppresseurs, en particulier de nature biologique, tels que des anticorps dirigés contre certains récepteurs des cellules lymphocytaires T, peut favoriser l'induction de tolérance.

D'autres éléments sont impliqués dans l'induction d'une tolérance: la forme physicochimique de l'antigène (l'élimination des agrégats dans une préparation d'immunoglobulines hétérologues, notamment, oriente la réponse immunitaire vers la tolérance [3]), la voie d'administration (la tolérance obtenue est meilleure par voie intraveineuse que par voie sous-cutanée), l'absence d'adjuvant (qui favorise la tolérance) et, enfin, la dose administrée (de façon générale, la tolérance dans les modèles expérimentaux est d'autant plus aisée à induire et durable que la dose d'antigène est élevée).

Pour affirmer l'existence d'une tolérance, il faut montrer que la paralysie immunitaire est bien spécifique du tolérogène, l'individu restant normalement répondeur vis-à-vis de tout autre antigène exogène. Cela n'est pas toujours techniquement facile, voire possible, en particulier lorsque la structure moléculaire du (ou des) tolérogène(s) n'est pas connue avec précision, comme c'est le cas de la tolérance immunitaire induite vis-à-vis de cellules allogéniques ou de greffes d'organe. On parlera alors de tolérance «opérationnelle », un individu présentant un état de tolérance opérationnelle vis-à-vis d'une allogreffe lorsque l'on constate une absence de toute réponse pathogène vis-à-vis de l'organe greffé après un traitement de durée limitée, et en l'absence d'une dépression globale des réponses immunitaires à long terme, telle que l'induit l'administration chronique d'immunosuppresseurs.

\section{La tolérance au soi et sa rupture: l'origine des maladies auto-immunes}

Le système immunitaire ne développe normalement pas de réaction agressive vis-à-vis des tissus de l'hôte qui l'héberge : c'est ce qu'il est convenu d'appeler la tolérance immunitaire physiologique. Néanmoins, tout sujet sain, indemne de pathologie auto-immune, héberge des lymphocytes autoréactifs dirigés contre divers autoantigènes, ou antigènes du «soi ». L'existence de ces lymphocytes autoréactifs est attestée par la présence d'auto-anticorps naturels et par la possibilité expérimentale d'induire des lignées ou des clones de lymphocytes T autoréactifs à partir de sang humain normal, ou même de provoquer une maladie auto-immune par l'administration d'autoantigènes à des animaux normaux, n'ayant pas de prédisposition particulière à développer une pathologie auto-immune spontanée. L'absence de maladies auto-immunes malgré la présence de lymphocytes B et T autoréactifs est un paradoxe central de l'immunologie, dont les mécanismes sont d'autant plus importants à analyser que c'est la rupture de cette tolérance au soi qui est responsable de l'émergence des maladies auto-immunes. Plusieurs mécanismes, non mutuellement exclusifs, ont été proposés.
Les lymphocytes autoréactifs les plus dangereux, car porteurs de récepteurs de très haute affinité pour les autoantigènes, sont en règle générale détruits dans les organes lymphoïdes centraux où ils sont produits (le thymus pour les lymphocytes $T$, la moelle osseuse pour les lymphocytes B). Ce phénomène de sélection «négative», qui implique une mort cellulaire par apoptose des lymphocytes autoréactifs concernés, est responsable de la «tolérance centrale ». Le filtre de la sélection négative qui concerne les autoantigènes spécialement représentés dans le thymus et la moelle osseuse est cependant loin d'être parfait, puisque l'on retrouve des lymphocytes autoréactifs à la périphérie. II existe des mécanismes, que l'on regroupe sous la dénomination de mécanismes de «tolérance périphérique », qui permettent de contrôler le potentiel pathogène des effecteurs autoréactifs ayant échappé à la sélection négative. Cet article est consacré plus particulièrement aux mécanismes de tolérance périphérique touchant les lymphocytes $T$, tout en sachant que des phénomènes identiques sont opérationnels pour les lymphocytes $B$.

Les cellules T autoréactives peuvent ne pas «reconnaître » les cellules présentant les autoantigènes, dont elles portent les récepteurs spécifiques, par un phénomène dit «d'indifférence immunitaire ». Cette indifférence est probablement due, au moins en partie, au fait que la plupart des cellules de l'organisme qui portent les autoantigènes ne les «présentent » pas de manière adéquate, ou «professionnelle», aux cellules immunitaires. En effet, seules les cellules professionnelles de la présentation de l'antigène, c'est-à-dire les cellules dendritiques, sont en mesure d'apporter aux lymphocytes $T$ l'ensemble des signaux indispensables à leur activation. Ainsi, un état d'indifférence peut être rompu lorsque l'autoantigène est présenté aux lymphocytes T d'une façon anormale, notamment lorsque les cellules cibles sont localisées au sein d'un site inflammatoire, par exemple induit par une infection virale.

Mais comment expliquer que la seule thymectomie réalisée chez une souris normale dans les jours suivant sa naissance induise un syndrome poly-auto-immun [4] ? II est apparu que cette thymectomie précoce prévient la dissémination à la périphérie d'une catégorie fonctionnelle particulière de lymphocytes $T$, aux propriétés régulatrices qui contrôlent l'autoréactivité physiologique. L'auto-immunité peut donc découler d'un défaut ou d'un débordement de mécanismes d'immunorégulation qui modulent l'intensité des réponses immunitaires. L'existence d'une telle immunorégulation est désormais bien prouvée. Elle fait intervenir les cellules T caractérisées par l'expression du marqueur CD25 (la chaîne $\alpha$ du récepteur de l'interleukine-2), souvent appelées Treg, et qui sont d'origine thymique : ce sont ces cellules dont il était question, ci-dessus, dans le cas de la thymectomie. II existe également une pléiade d'autres cellules T régu- 
latrices telles que les cellules Th2 (productrices d'IL-4 et d'IL-10), les cellules exprimant la L-sélectine (CD62L'), les cellules Trl (productrices d'IL-10 et de TGF $\beta$ (transforming growth factor $\beta$ ), les cellules Th3 (productrices de TGF $\beta$ ), les cellules $T \gamma \delta$ et, enfin, les lymphocytes invariants NKT (natural killer T cell) $[5,6]$.

Quel que soit le mécanisme sous-jacent à l'éclosion de la réponse auto-immune pathogène, rupture de l'indifférence immunitaire ou défaillance de l'immunorégulation, la question est de savoir si la restauration de la tolérance au soi ne pourrait pas contribuer favorablement au traitement des maladies auto-immunes en tant qu'approche thérapeutique. Cela éviterait le recours aux médicaments immunosuppresseurs conventionnels, non spécifiques de l'antigène, avec leur cortège d'effets secondaires directs (comme la néphrotoxicité de la ciclosporine ou la myélotoxicité et la carcinogénicité du cyclophosphamide) ou liés à la «surimmunosuppression» engendrée par leur administration chronique, et conduisant à une incidence augmentée d'infections et de tumeurs (souvent des lymphomes liés à des réactivations virales mal contrôlées, lymphomes induits par le virus d'Epstein Barr).

Dans le cas du diabète insulinodépendant, ou diabète de type 1 , deux stratégies sont en cours de développement clinique dans ce but. La première consiste en l'administration des autoantigènes cibles sous forme «tolérogène ». La seconde repose sur l'utilisation d'anticorps monoclonaux anti-cellules $T$, et plus particulièrement celle d'anticorps dirigés contre la molécule CD3.

\section{Les tentatives de restauration de la tolérance par l'administration d'autoantigènes solubles}

Le diabète insulinodépendant résulte de la destruction sélective des cellules $\beta$ des îlots de Langerhans, productrices d'insuline, par des lymphocytes T autoréactifs $\mathrm{CD}^{+}$et $\mathrm{CD}^{+}[7]$. Notre connaissance actuelle des mécanismes physiopathologiques de la maladie a grandement bénéficié de l'étude de modèles animaux tels que la souris NOD (non obese diabetic) qui développe spontanément un diabète auto-immun.

L'administration de certains antigènes présents dans les cellules $\beta$ des îlots de Langerhans, tels que l'insuline, la décarboxylase de l'acide glutamique (GAD) ou la protéine de choc thermique hsp60, induit chez la jeune souris NOD un état de tolérance qui prévient l'apparition du diabète, habituellement observée entre 3 à 9 mois d'âge [8-11]. Cette tolérance fait intervenir, pour l'essentiel, la différenciation de cellules Th2, spécifiques de l'autoantigène administré, productrices d'IL-4 et d'IL-10 [10, 12].

Cette stratégie est logique. Elle a l'avantage thérapeutique d'être dispensée de toxicité, notamment du risque de surimmunosuppression, même si certains résultats (rapportés au cours d'essais effectués dans la sclérose en plaques) ont indiqué la survenue d'épisodes d'anaphylaxie, voire d'aggravation de la maladie plutôt que l'effet curatif espéré [13].

En tout état de cause, même si les études de phase I/II menées dans cet esprit avaient suggéré des tendances encourageantes [14-17], aucun des essais cliniques contrôlés engagés par la suite n'a donné de résultats probants $[13,18-20]$, exception faite d'un essai utilisant le peptide p277, issu de la protéine de choc thermique hsp 60, chez des patients présentant un diabète de type 1 de diagnostic récent; les résultats de cet essai doivent cependant être confirmés [21]. De fait, qu'il s'agisse de la voie orale, pourtant très prometteuse dans les modèles expérimentaux de peptides modifiés (APL, altered peptide ligand [22]) pour réduire leur immunogénicité, des fragments peptidiques des autoantigènes ou de la molécule entière, les résultats obtenus dans le diabète de type 1, mais aussi dans d'autres pathologies auto-immunes telles que la sclérose en plaques ou les uvéites, se sont révélés pour l'instant négatifs, ou peu concluants. Cette déception peut trouver plusieurs explications : traitement de malades à un stade trop avancé de la maladie, doses d'autoantigène administré insuffisantes (surtout par rapport à celles utilisées chez la souris). Des essais sont toujours en cours, notamment dans le diabète, qui tentent de surmonter ces difficultés.

\section{L'effet surprenant des anticorps anti-CD3 chez la souris NOD présentant un diabète déclaré}

Dans le prolongement des expériences réalisées en immunité de transplantation avec les anticorps monoclonaux anti-T mentionnés plus haut, il était tentant de restaurer la tolérance au soi dans les maladies autoimmunes avec l'un des plus puissants de ces anticorps, l'anticorps antiCD3. La molécule CD3 est en fait un complexe moléculaire étroitement associé au récepteur des cellules T pour la reconnaissance de l'antigène, dont il représente l'élément de transduction du signal, délivré lors de la reconnaissance du peptide antigénique présenté par la molécule d'histocompatibilité à la surface de la cellule présentatrice de l'antigène [23]. De manière intéressante, l'administration isolée d'anticorps anti-CD3 à de jeunes souris NOD, à l'âge où les autoantigènes solubles sont protecteurs, ne prévient pas la survenue du diabète [24]. En revanche, et de façon totalement surprenante, un traitement de courte durée (seulement cinq jours) de souris NOD récemment devenues diabétiques induit une rémission complète et définitive de la maladie [25]. La rémission est déclenchée par la disparition de l'infiltrat de cellules T des îlots (ou insulite), une disparition toutefois transitoire, qui n'est donc pas impliquée dans l'effet thérapeutique à long terme. Dès 2 semaines après la fin du traitement, l'insulite récidive mais sous forme d'un infiltrat périphérique qui n'envahit pas les îlots et ne détruit pas les cellules $\beta$ résiduelles $[25,26]$. L'effet thérapeutique initial est relayé par la différenciation de cellules T régulatrices, dont nous avons montré qu'elles dépendaient étroitement de la production du TGF $\beta[26,27]$. L'anticorps induit bien un état de tolérance (au moins opérationnelle), puisque les lymphocytes T des souris protégées du diabète ne répondent pas de manière pathogène vis-à-vis des antigènes d'îlots, tandis qu'elles récupèrent une capacité absolument normale de rejeter 
des greffes de peau allogéniques [25]. Cette tolérance est bien «active», c'est-à-dire dépendante de cellules T régulatrices, puisqu'elle est abrogée par l'administration de cyclophosphamide [24, 26], un agent alkylant connu pour son effet sélectif sur les lymphocytes T régulateurs [28, 29]. L'induction d'un tel état de tolérance par l'administration d'un anticorps sans spécificité pour les autoantigènes des cellules $\beta$ peut surprendre. Sans doute l'anticorps agit-il sélectivement sur les cellules T activées qui prédominent dans les îlots chez ces souris, ce qui lui confère sa spécificité. Cette interprétation explique aussi l'absence d'efficacité de l'anticorps chez les jeunes souris, dont les cellules T infiltrant les îlots sont peu ou pas activées (à ce stade, l'insulite n'est pas invasive ni destructrice).

Les anticorps anti-CD3 ont été largement utilisés chez l'homme dans les années 1980 pour le traitement et la prévention du rejet d'allogreffes d'organes, sous la forme de l'OKT3 [30,31]. L'efficacité en avait été remarquable, mais l'activation des cellules $T$ et la libération massive de cytokines qui en découlait avaient limité son utilisation et empêché son application aux affections auto-immunes [26, 32, 33]. Les résultats décrits ci-dessus obtenus chez la souris NOD, qui ont été reproduits dans d'autres modèles expérimentaux tels que l'encéphalomyélite allergique expérimentale [34] et la colite inflammatoire [35], ont constitué le rationnel préclinique pour étendre l'application des anticorps anti-CD3 à l'auto-immunité. Cela a par ailleurs été rendu possible par la disponibilité d'anticorps anti-CD3 humanisés par génie génétique, et présentant des mutations dans leur fragment Fc (la partie constante des anticorps) empêchant leur fixation aux macrophages, une étape indispensable à l'expression de leurs propriétés d'activation $[26,36,37]$. Ces anticorps anti-CD3 humanisés sont donc beaucoup moins activateurs que l'OKT3, et de ce fait bien tolérés en clinique $[38,39]$. Le champ était ainsi ouvert pour l'utilisation des anticorps anti-CD3 dans les maladies auto-immunes chez l'homme. C'est ce qui a été réalisé avec succès dans le diabète de type 1 .

\section{Les anticorps anti-CD3 induisent une rémission durable du diabète humain établi}

Les résultats d'une étude de phase I utilisant l'anticorps OKT3 $\gamma 1$ Ala-Ala chez 12 patients traités et 12 sujets contrôles non traités [40] ont confirmé la bonne tolérance du produit, tout en suggérant la présence d'un effet thérapeutique favorable un an après le traitement [40]. Cette tendance a été confirmée par le suivi à deux ans d'un nombre plus important de patients [41]. En collaboration avec des cliniciens et des biologistes belges, allemands et anglais, nous avons conduit une étude de phase II contrôlée, randomisée contre placebo, incluant un total de 80 patients et utilisant un autre anticorps humanisé, ChAglyCD3 [42]. L'anticorps ou le placebo ont été administrés pendant 6 jours consécutifs ( $8 \mathrm{mg} /$ jour). Le traitement préserva très efficacement la production d'insuline endogène (mesurée par le taux de peptide $C$ après stimulation par du glucose intraveineux) 6, 12 et même 18 mois après le traitement. Une diminution significative des doses d'insuline exogène a également été observée [42]. De manière remarquable, à 18 mois, $75 \%$ des patients traités par l'anticorps qui présentaient au début du traitement une masse de cellules $\beta$ plus importante montraient des besoins en insuline inférieurs ou égaux à $0,25 \mathrm{U} / \mathrm{kg} / \mathrm{jour}$, ce qui est une dose proche de l'insulino-indépendance [42].
Concernant les effets secondaires, nous avons observé une libération de cytokines modérée, survenant après les premières injections et provoquant des symptômes limités. En deuxième lieu, les patients ont montré, deux à trois semaines après le traitement, une réactivation transitoire du virus d'Epstein-Barr ( $\varepsilon B V)$, accompagnée de signes cliniques passagers de mononucléose infectieuse. La réaction s'est éteinte en 2 à 3 semaines, avec le retour à la normale des copies d'EBV (devenues indétectables) du fait de l'apparition d'une réponse immunitaire humorale et cellulaire, spécifique de l'£BV, efficace [42]. Cette réponse anti-દBV est, pour des raisons évidentes, très importante du point de vue de la bonne tolérance de l'anticorps. Elle atteste, par ailleurs, d'une situation proche de celle observée chez la souris, où l'anticorps anti-CD3 semble induire un effet spécifique des autoantigènes d'îlots, puisqu'un effet durable est observé sur le diabète, la maladie auto-immune, alors que les patients sont répondeurs, très rapidement après la fin $\mathrm{du}$ traitement, vis-à-vis d'autres antigènes tels que l'£BV.

\section{Conclusions}

La possibilité qu'offrent les anticorps anti-CD3 de restaurer la tolérance vis-à-vis des antigènes du soi représente une étape importante du développement de l'immunothérapie des maladies auto-immunes, qui ouvre de très nombreuses perspectives.

Au niveau pharmacologique, la recherche sur les anticorps anti-CD3 va probablement susciter un intérêt croissant pour le développement d'agents permettant d'augmenter le nombre ou de stimuler la capacité fonctionnelle de cellules $T$ régulatrices. Les données expérimentales indiquent clairement que les anticorps anti-CD3 suscitent un effet thérapeutique qui est spécifique de l'antigène par leur capacité à stimuler sélectivement des cellules $T$ régulatrices capables de contrôler localement, au niveau de l'organe cible, les effecteurs pathogènes.

Du point de vue thérapeutique, les anticorps anti-CD3 pourraient permettre à l'avenir de réduire l'administration chronique d'immunosuppresseurs chimiques, qui constituent encore le traitement de base de nombreuses pathologies auto-immunes. Dans ce contexte, les résultats spectaculaires obtenus dans le traitement du diabète insulino-dépendant de diagnostic récent sont extrêmement encourageants. Ces résultats peuvent certainement être encore améliorés en étant plus strict sur la sélection des patients et, peut-être, en tirant partie d'associations thérapeutiques (notamment avec des autoantigènes solubles).

Enfin, au vu des résultats expérimentaux disponibles, I'utilisation des anticorps anti-CD3 pourrait s'étendre rapidement à d'autres indications en auto-immunité, ainsi qu'en transplantation. $\diamond$ 


\section{SUMMARY}

One step towards restoration of self-tolerance

in human autoimmune diseases

In developed countries the incidence of autoimmune insulin-dependent or type 1 diabetes as the one of all autoimmune diseases has steadily increased over the last decades. Conventional therapy of type 1 diabetes is essentially palliative namely, chronic delivery of exogenous insulin that is associated with major constraints (multiple daily parenteral administration, serious risks linked to hypoglycemic episodes) and incomplete effectiveness in preventing severe degenerative complications. This explains the growing attention on modern therapeutic strategies using biological agents such as CD3 monoclonal antibodies that allow «reprogramming » the immune system to restore self-tolerance to pancreatic beta cell antigens. This strategy which proved successful in the experimental setting has recently been translated to the clinic with very encouraging results. CD3 antibodies may represent a new category of drugs affording a real cure for autoimmunity namely, inhibiting the pathogenic immune response while preserving the host reactivity to unrelated antigens. $\diamond$

\section{RéFÉRENCES}

1. Billingham RE, Brent L, Medawar PB. Actively acquired tolerance to foreign cells. Nature $1953 ; 172$ : 603-6.

2. Brent L, Courtenay T, Gowland G. Immunological reactivity of lymphoid cells after treatment with anti-lymphocytic serum. Nature 1967 ; 215: 1461-4.

3. Dresser DW. Specific inhibition of antibody production. II. Paralysis induced in adult mice by small quantities of protein antigen. Immunology $1962 ; 5: 378-88$.

4. Sakaguchi S. Naturally arising $C D 4^{+}$regulatory T cells for immunologic self-tolerance and negative control of immune responses. Annu Rev Immunol $2004 ; 22$ : 531-62.

5. Bach JF. Regulatory T cells under scrutiny. Nat Rev Immunol 2003; 3: 189-98.

6. Chatenoud L, Salomon B, Bluestone JA. Suppressor T cells. They're back and critical for regulation of autoimmunity! Immunol Rev 2001 ; 182: 149-63.

7. Bach JF. Insulin-dependent diabetes mellitus as an autoimmune disease. Endocrine Rev 1994 ; 15 : 516-42.

8. Kaufman DL, Clare-Salzler M, Tian J, et al. Spontaneous loss of T-cell tolerance to glutamic acid decarboxylase in murine insulin-dependent diabetes. Nature $1993 ; 366: 69-72$.

9. Tisch R, Yang X D, Singer SM, et al. Immune response to glutamic acid decarboxylase correlates with insulitis in non-obese diabetic mice. Nature $1993 ; 366: 72-5$.

10. Elias D, Meilin A, Ablamunits V, et al. Hsp60 peptide therapy of NOD mouse diabetes induces a Th2 cytokine burst and downregulates autoimmunity to various beta-cell antigens. Diabetes $1997 ; 46: 758-64$.

11. Tian J D, Claresalzler M, Herschenfeld A, et al. Modulating autoimmune responses to $G A D$ inhibits disease progression and prolongs islet graft survival in diabetes-prone mice. Nat Med 1996; 2 : 1348-53.

12. Tian J, Lehmann PV, Kaufman DL. Determinant spreading of Thelper cell 2 (Th2) responses to pancreatic islet autoantigens. J Exp Med 1997 ; $186: 2039-43$.

13. Sospedra M, Martin R. Antigen-specific therapies in multiple sclerosis. Int Rev Immunol 2005 ; 24 : 393-413.

14. Weiner HL, Mackin GA, Matsui M, et al. Double-blind pilot trial of oral tolerization with myelin antigens in multiple sclerosis. Science 1993 ; 259: 1321-4
15. Barnett ML, Kremer JM, St Clair EW, et al. Treatment of rheumatoid arthritis with oral type II collagen. Results of a multicenter, double-blind, placebo-controlled trial. Arthritis Rheum 1998 ; $41: 290-7$.

16. Barnett ML, Combitchi D, Trentham DE. A pilot trial of oral type II collagen in the treatment of juvenile rheumatoid arthritis. Arthritis Rheum 1996 ; $39: 623-8$.

17. Nussenblatt RB, Gery I, Weiner HL, et al. Treatment of uveitis by oral administration of retinal antigens : Results of a phase I/II randomized masked trial. Am J Ophthalmol 1997 ; 123 : 583-92.

18. Chaillous $L$, Lefevre $H$, Thivolet $C$, et al. Oral insulin administration and residual beta-cell function in recent-onset type 1 diabetes: a multicentre randomised controlled trial. Diabete insuline orale group. Lancet $2000 ; 356: 545-9$.

19. Ergun-Longmire B, Marker J, Zeidler A, et al. Oral insulin therapy to prevent progression of immunemediated (type 1) diabetes. Ann NY Acad Sci $2004 ; 1029: 260-77$.

20. Skyler J. Effects of insulin in relatives of patients with type 1 diabetes mellitus. Diabetes prevention trial-type 1 diabetes study group. N Engl J Med 2002 ; 346 : 1685-91.

21. Raz I, Elias D, Avron A, et al. Beta-cell function in new-onset type 1 diabetes and immunomodulation with a heat-shock protein peptide (DiaPep277): a randomised, double-blind, phase II trial. Lancet $2001 ; 358: 1749-53$.

22. Brocke S, Gijbels K, Allegretta M, et al. Treatment of experimental encephalomyelitis with a peptide analogue of myelin basic protein. Nature 1996 ; 379: 343-6.

23. Clevers $H$, Alarcon B, Wileman T, et al. The T cell receptor/CD3 complex: a dynamic protein ensemble. Annu Rev Immunol 1988; $6: 629-62$.

24. Chatenoud L, Primo J, Bach J F. CD3 antibody-induced dominant self tolerance in overtly diabetic NOD mice. J Immunol $1997 ; 158: 2947-54$.

25. Chatenoud L, Thervet $\varepsilon$, Primo J, et al. Anti-CD3 antibody induces long-term remission of overt autoimmunity in nonobese diabetic mice. Proc Natl Acad Sci USA $1994 ; 91$ : 123-7.

26. Chatenoud L. CD3-specific antibody-induced active tolerance: from bench to bedside. Nat Rev Immunol $2003 ; 3: 123-32$.

27. Belghith M, Bluestone JA, Barriot S, et al. TGF-beta-dependent mechanisms mediate restoration of self-tolerance induced by antibodies to CD3 in overt autoimmune diabetes. Nat Med 2003; $9: 1202-8$.

28. Yasunami R, Bach JF. Anti-suppressor effect of cyclophosphamide on the development of spontaneous diabetes in NOD mice. Eur J Immunol 1988; $18: 481-4$.

29. Mahiou J, Walter U, Lepault F, et al. In vivo blockade of the Fas-Fas ligand pathway inhibits cyclophosphamide-induced diabetes in NOD mice. J Autoimmun $2001 ; 16: 431-40$.

30. Cosimi AB, Colvin RB, Burton RC, et al. Use of monoclonal antibodies to T-cell subsets for immunologic monitoring and treatment in recipients of renal allografts. $N$ Engl J Med 1981 ; 305 : 308-14.

31. Ortho multicenter transplant study group. A randomized clinical trial of OKT3 monoclonal antibody for acute rejection of cadaveric renal transplants. N Engl J Med $1985 ; 313: 337-42$.

32. Chatenoud L, Ferran C, Legendre C, et al. In vivo cell activation following OKT3 administration. Systemic cytokine release and modulation by corticosteroids. Transplantation 1990; $49: 697-702$.

33. Abramowicz D, Schandene L, Goldman M, et al. Release of tumor necrosis factor, interleukin-2, and gamma-interferon in serum after injection of OKT3 monoclonal antibody in kidney transplant recipients. Transplantation $1989 ; 47: 606-8$.

34. Kohm AP, Williams JS, Bickford AL, et al. Treatment with nonmitogenic anti-CD3 monoclonal antibody induces $C D 4+T$ cell unresponsiveness and functional reversal of established experimental autoimmune encephalomyelitis. J Immunol 2005 ; $174: 4525-34$.

35. Ludviksson BR, Ehrhardt RO, Strober W. TGF-beta production regulates the development of the 2,4,6-trinitrophenol-conjugated keyhole limpet hemocyanin-induced colonic inflammation in IL2-deficient mice. J Immunol 1997 ; 159 : 3622-8.

36. Alegre ML, Peterson LJ, Xu D, et al. A non-activating «humanized » anti-CD3 monoclonal antibody retains immunosuppressive properties in vivo. Transplantation $1994 ; 57: 1537-43$.

37. Bolt S, Routledge $\varepsilon$, Lloyd I, et al. The generation of a humanized, non-mitogenic CD3 monoclona antibody which retains in vitro immunosuppressive properties. Eur J Immunol 1993 ; 23: 403-11.

38. Woodle ES, Xu D, Zivin RA, et al. Phase I trial of a humanized, Fc receptor nonbinding OKT3 antibody, hu0KT3gammal (Ala-Ala) in the treatment of acute renal allograft rejection. Transplantation $1999 ; 68: 608-16$.

39. Friend PJ, Hale G, Chatenoud L, et al. Phase I study of an engineered aglycosylated humanized CD3 antibody in renal transplant rejection. Transplantation 1999; 68 : 1632-7.

40. Herold KC, Hagopian W, Auger JA, et al. Anti-CD3 monoclonal antibody in new-onset type 1 diabetes mellitus. $N$ Engl J Med 2002 ; 346 : 1692-8.

41. Herold KC, Gitelman SE, Masharani U, et al. A single course of anti-CD3 monoclonal antibody hOKT3gammal (Ala-Ala) results in improvement in C-peptide responses and clinical parameters for at least 2 years after onset of type 1 diabetes. Diabetes $2005 ; 54: 1763-9$.

42. Keymeulen $B$, Vandemeulebroucke $\varepsilon$, Ziegler A G, et al. Insulin needs after CD3-antibody therapy in new-onset type 1 diabetes. $N$ Engl J Med 2005 ; 352: 2598-608. 


\section{Ateliers de formation 2007}

Renseignements et inscriptions :

Ateliers de formation Inserm

101, rue de Tolbiac

75654 Paris Cedex 13

Tél. : 33 (0) 144236203 - Fax : 33 (0) 144236293

ateliers@tolbiac.inserm.fr

\section{Inserm \\ Institut national \\ de la santé et de la recherche médicale}

\section{Atelier de formation $n^{\circ} 181$}

\section{Cribles génomiques par interférence par l'ARN dans des cellules en culture}

Organisateurs : François Dautry (CNRS-FRE 2937, Villejuif), Jean Imbert (U599 Inserm, Marseille), Jean-Jacques Lawrence, Représentant de I'Inserm aux États-Unis (Ambassade de France, Washington, DC)

\section{Phase I • Le point sur... \\ 11-12 juin 2007• La Londe-Les-Maures (Toulon)}

Objectifs - Le concept de régulation de l'expression génétique par l'intervention de molécules d'ARN double-brin est maintenant bien établi. II existe différentes voies possibles pour l'extinction de l'expression de gènes spécifiques par diverses formes d'ARN double brin, et les mécanismes impliqués sont en bonne voie de caractérisation. L'importance de tels mécanismes de régulation s'est rapidement imposée, en raison à la fois de leur intérêt biologique, mais aussi du potentiel qu'ils représentent au plan expérimental, et, peut-être, à l'avenir, thérapeutique. Deux Ateliers Inserm ont eu lieu sur des aspects particuliers de ce thème: ARN interférence et génomique (2004) et micro RNA et régulation de l'expression génétique (2006). L'organisation d'un troisième Atelier sur ce même thème à une date aussi rapprochée est motivée par deux arguments : 1) l'accélération du sujet en raison en particulier de l'impact de la génomique : la connaissance de génomes entiers permet d'envisager des études au niveau du génome et plus seulement de quelques gènes, ceci s'inscrivant dans la continuité d'autres démarches comme les études du transcriptome, du protéome et de l'interactome ; 2) l'opportunité qui est donnée d'organiser la phase pratique de cet Atelier avec le support de la «RNAi High-Throughput Screening (HTS) platform », plateforme nationale des NIH, animée par le Dr. Norbert Perrimon au sein de la Harvard School of Medicine (Drosophila RNAi Screening Center: http://flyrnai.org/).

Public - Chercheurs, médecins, techniciens, ingénieurs, postdocs et étudiants intéressés par l'utilisation de l'interférence pour identifier les gènes impliqués dans un processus physiologique ou pathophysiologique donné.

Les conférences seront en anglais.

Programme • I - Rappels : Nature et mécanismes d'action des ARN interférant. II - Les cribles à grande échelle, présentation générale. III - Les outils d'analyse et de traitement de l'information. IV - Exemples de cribles à grande échelle. V - In vivo RNAi. VI - Table ronde sur les principales avancées de la biologie systémique.

Nombre maximum de participants : 80 .

Avec la participation pressentie de • Julie Ahringer (Cambridge, Grande Bretagne), Roderick Beijersbergen (Amsterdam, Pays Bas), François Dautry (Villejuif, France), Christophe Echeverri (Dresde, Allemagne), Xavier Gidrol (Évry, France), Annick Harel-Bellan (Villejuif, France), Aimee Jackson (Seattle, USA), Ihor Lemischka (Princeton, USA), Bernard Mathey-Prevot (Boston, USA), Norbert Perrimon (Boston, USA), Jussi Taipale (Helsinki, Finlande), Marc Vidal (Boston, USA).

\section{Phase II • Maîtrise technique}

Septembre 2007 (6 jours) • Boston (Harvard Medical School)

Le stage pratique ayant lieu aux États-Unis, son coût sera pris en charge partiellement par la Mission pour la Science et la Technologie de l'Ambassade de France.

Programme • Initiation aux criblages de génomique fonctionnelle sur la plateforme DRSC de Harvard Medical School. Le programme détaillé de la phase II sera communiqué avec l'appel à projet qui servira à la sélection des candidats.

Sélection $\bullet 4$ stagiaires maximum seront sélectionnés parmi les participants de la phase I sur la base de leur projet scientifique et des perspectives d'utilisation de cette approche dans leur laboratoire d'accueil.

Date limite d'inscription : 11 avril 2007 